\title{
Auto-inhibitory effects of an IQ motif on protein structure and function
}

Daria Petzhold ${ }^{1}$, Janine Lossie $^{1}$, Joachim Behlke ${ }^{1}$, Sandro Keller ${ }^{3}$, Hannelore Haase ${ }^{1}$, Ingo Morano ${ }^{1,2}$

${ }^{1}$ Max-Delbrück-Center for Molecular Medicine, Dept. Molecular Muscle Physiology, Robert-Rössle-Strasse 10, 13125 Berlin, Germany;

${ }^{2}$ University Medicine Charité Berlin, Charitéplatz 1, 10117 Berlin, Germany,

${ }^{3}$ Molecular Biophysics University of Kaiserslautern, Erwin-Schrödinger-Str. 13,

67663 Kaiserslautern, Germany

Correspondence to:

Daria Petzhold, PhD.

Max Delbrück Center for Molecular Medicine (MDC)

Robert-Rössle-Str. 10

D-13092 Berlin, Germany

Tel.: ++49-30-9406-2313; Fax: ++49-30-9406-2579

Email: petzhold@mdc-berlin.de

Email addresses of the authors:
Janine Lossie: $\quad$ janine.lossie@mdc-berlin.de
Joachim Behlke: $\quad$ behlke@mdc-berlin.de
Sandro Keller: $\quad$ sandro.keller@biologie.uni-kl.de
Hannelore Haase: haase@mdc-berlin.de
Ingo Morano: $\quad$ imorano@mdc-berlin.de 


\section{Abstract}

The denuded IQ2 domain, i.e. myosin heavy chain not associated with regulatory light chains, exerts an inhibitory effect on myosin ATPase activity. In this study, we elaborated a structural explanation for this auto-inhibitory effect of IQ2 on myosin function. We employed analytical ultracentrifugation, circular dichroism, and surface plasmon resonance spectroscopy to investigate structural and functional properties of a myosin heavy chain (MYH) head-rod fragment aa664-915. $\mathrm{MYH}_{664-915}$ was monomeric, adopted a closed shape, and bound essential myosin light chains (HIS-MLC-1) with low affinity to IQ1. Deletion of IQ2, however opened $\mathrm{MYH}_{664-915}$. Four amino acids present in IQ2 could be identified to be responsible for this auto-inhibitory structural effect: alanine mutagenesis of 1814, Q815, R819, and W827 stretched $\mathrm{MYH}_{664-915}$ and increased 30 -fold the binding affinity of HIS-MLC-1 to IQ1. In this study we show, that denuded IQ2 favours a closed conformation of myosin with a low HIS-MLC-1 binding affinity. The collapsed structure of myosin with denuded IQ2 could explain the auto-inhibitory effects of IQ2 on enzymatic activity of myosin.

\section{Keywords}

IQ motif; myosin; myosin light chains; analytical ultracentrifugation; protein structure; Protein-protein-interaction 


\section{Introduction}

Many cellular proteins including neuronal growth proteins, myosins, voltageoperated ion channels, phosphatases, Ras exchange proteins, sperm surface proteins, a Ras Gap-like protein, spindle-associated proteins and several proteins in plant contain IQ motifs for $\mathrm{Ca}^{2+}$-dependent and independent interaction with calmodulin and calmodulin-like proteins, e.g. myosin light chains, thus regulating a wide diversity of biological functions [1]. Myosin II consists of two heavy chains (MYH) each associated with two light chains (MLC). It is the motor protein of muscle contraction which interacts cyclically with the thin (actin) filament, thus producing force and shortening while consuming ATP. Each MYH can be separated into: (i) an N-terminal "head" or subfragment 1 (S-1) (aa1-837) consisting of a pear-shaped $8 \mathrm{~nm}$ motor domain with ATPase and actin binding sites, connected to the regulatory domain, i.e. an $\alpha$-helical $8.5 \mathrm{~nm}$ lever arm which binds myosin light chains; (ii) a $150 \mathrm{~nm}$ a-helical rod (aa838-1935), consisting of a $60 \mathrm{~nm}$ subfragment 2 (S2), and a C-terminal $90 \mathrm{~nm}$ light meromyosin (LMM) (Figure 1). S-2 and LMM form a coiled-coil with a second MYH $[2,3]$ (numbers for MYH7, i.e. rat cardiac $\beta$-MYH, accession NP_058936, used throughout in this study). The lever arm of myosin II contains two IQ motifs in tandem, designated as IQ1 (IQAQSRGVLSRMEFKKL, aa788-802) which forms a complete (consensus [FILV]Qxxx[RK]Gxxx[RK]xx[FILVWY] in one-letter amino acid code, where $x$ is any amino acid and brackets indicate an alternative), and IQ2 (IQWNIRAFMGVKNWPWM, aa814-828) which forms an incomplete (consensus [FILV]Qxxx[RK]xxxxxxxx) [4] IQ motif. IQ1 of the lever arm binds 
essential MLCs (ELC, HIS-MLC-1), IQ2 binds regulatory MLCs (RLC, MLC-2) [2-5].

\section{Material and Methods}

Generation of GST- and His-tag fusion proteins

Rat essential ventricular myosin light chain (MLC-1) and three headrod fragments were cloned with an N-terminal hexahistidine (His)-tag/40 amino acid (aa) spacer and an N-terminal glutathione S-transferase (GST)tag/14aa spacer, respectively.

To prepare the His-MLC-1 fusion protein rat cardiac mRNA was transcribed with random primers and used as templates. The region of the cDNA carrying the protein-coding of HIS-MLC-1 was amplified using specific primers. HindIII restriction sites were incorporated into primers to facilitate subsequent subcloning into the HindIII site of the expression vector pRSETA (Invitrogen $\mathrm{GmbH}$, Karlsruhe, Germany).

Likewise, the head-rod fragment aa664-915 of rat cardiac $\beta-\mathrm{MYH}$ (accession NP_058936; MYH7) was amplified using specific primers from rat cardiac cDNA $\left(\mathrm{MYH}_{664-915}\right)$ and cloned into pGEX-4T1 expression vector (StratageneEurope, Amsterdam, Netherlands) (GST-MYH $664-915)$. N-terminal GST-tag was used to allow interactions of its C-terminal IQ domains with the MLC.

The consensus sequence IQWNIRAFMGVKNW (aa814-828) of IQ2 in GST$\mathrm{MYH}_{664-915}$ was deleted (GST-MYH $\mathrm{G64-915}^{\mathrm{LI} 2}$ ) in pGEX-4T1 by introducing the restriction sides Stul upstream and downstream of the IQ2 motif using the QuickChange site-directed mutagenesis kit (StratageneEurope, Amsterdam, 
Netherlands) and subsequently digested with Stul. Following removal of the IQ2 DNA fragment the vector was re-ligated and transformed into TOP10 and BL21 (DE3) pLysS cells, respectively.

The IQ2 motif of GST-MYH $\mathrm{H}_{664-915}$ was deteriorated by alanine site-directed mutagenesis $\left(\right.$ GST-MYH ${ }_{664-915}{ }^{\text {IQala4}}$ ). Alanine was used as the substituting amino acid because its small side chain would be expected to minimally perturb the structure of the protein. Four key amino acid residues of IQ2 known to be involved in RLC binding (I814, Q815, R819, W827) [4, 5] were replaced by alanine using the QuickChange site-directed mutagenesis kit (StratageneEurope, Amsterdam, Netherlands) according to the manufacturers protocol.

The pRSETA containing His-MLC-1 cDNA, and the pGEX-4T1 expression vectors containing the cDNAs of the head-rod constructs were used to transform BL21 (DE3) pLysS cells (Invitrogen GmbH, Karlsruhe, Germany). Protein expression was induced with $0.1 \mathrm{mM}$ isopropyl(-D)thiogalactopyranoside (IPTG; Diagnostic Chemicals Ltd.) for 3-4 h at $37^{\circ} \mathrm{C}$. Cells were then sonicated, centrifuged, and the supernatant prepared for purification of the different recombinant proteins: (1) HIS-MLC-1 was incubated with $0.5 \mathrm{ml}$ of Ni-NTA-agarose increased beads (Qiagen, Germany) and fusion protein was eluted with $100 \mathrm{mM}$ imidazole, $300 \mathrm{mM} \mathrm{NaCl}, 50 \mathrm{mM}$ $\mathrm{NaH}_{2} \mathrm{PO}_{4} \mathrm{pH}$ 8.0. (2) The head-rod proteins were incubated with glutathionesepharose beads for $10 \mathrm{~min}$ at room temperature. Fusion proteins were eluted with $20 \mathrm{mM}$ glutathione in $50 \mathrm{mM} \mathrm{NaHCO}$ buffer, $\mathrm{pH}$ 9.0, and dialyzed against PBS (100 mM NaCl, 1 mM EGTA, 5 mM Na $2 \mathrm{HPO}_{4}$ ), pH 7.4. 
Analysis of protein-protein interactions by surface plasmon resonance spectroscopy

Binding studies of the recombinant fusion proteins were carried out in a BIAcore 2000 Instrument (Uppsala Sweden) at $25^{\circ} \mathrm{C}$ using the sensor chip CM5 (BiAcore AB). Sensor chips were chemically activated by the injection of $90 \mu$ of a 1:1 mixture of N-hydroxysuccinimide (NHS, $100 \mathrm{mM}$ ) and N-ethylN'-(3dimethylaminopropyl)carbodi-imide (EDC, 400mM) at a flow rate of $10 \mu \mathrm{l} / \mathrm{min}$. For immobilization the recombinant protein HIS-MLC-1 was diluted in a $10 \mathrm{mM}$ acetate buffer, $\mathrm{pH} 4.5$, and was immobilized at a binding level of 2 $\mathrm{ng} / \mathrm{mm}^{2}$, which was based on the assumption that a surface plasmone resonance (SPR) response of 1000 relative units $(R U)$ translates to $1 \mathrm{ng} / \mathrm{mm}^{2}$ immobilized protein. Equimolar concentration of a synthetic His-tag/40aa spacer protein as control was immobilized on a separate lane of the same chip. The remaining matrix sites were blocked by the injection of $70 \mu \mathrm{l}$ of $1 \mathrm{M}$ ethanolamine, $\mathrm{pH}$ 8.5. Purified recombinant lever arms (GST-MYH $664-915$, GST-MYH $_{664-915^{\text {Iala4 }}}$, GST-MYH ${ }_{664-915}{ }^{\mathrm{IO} 2}$ ) diluted in PBS (100 mM NaCl, 1 mM EGTA, 5 mM Na2HPO4), pH 7.4 were used as analyte and were injected into the flow cells at a perfusion rate of $10 \mu \mathrm{l} / \mathrm{min}$. The analyte concentrations ranged from 0.125 to $15 \mu \mathrm{M}$. Between sample injections the surface was regenerated with $5 \mu \mathrm{l}$ of buffer containing $133 \mathrm{mM} \mathrm{NaCl}, 8 \mathrm{mM} \mathrm{NaOH}, 0.05 \%$ CHAPS, $0.05 \%$ Tween 80, 0.05\% Tween 20 and $0.05 \%$ Triton X-100. For data analysis, rate constants were calculated by global fitting using the BIAevaluation 3.2 RC 1 program (Biacore $A B$ ). Curves were fitted to a singlesite interaction model. Equilibrium $K_{D}$ values were determined from the rate 
constants $\mathrm{k}_{\text {on }}$ and $\mathrm{k}_{\text {off }}$ according to $\mathrm{K}_{\mathrm{D}}=\mathrm{k}_{\text {off }} / \mathrm{k}_{\text {on }}$. The analysis software corrects for systematic drift in baseline that occurred during measurements.

\section{Analytical ultracentrifugation}

Molecular mass studies on dissolved GST-MYH $\mathrm{H}_{664-915}$ and GST-MYH $664-$ $915^{\text {IQala4 }}$ were performed in a XL-A type analytical ultracentrifuge (Beckman, Palo Alto, CA, USA) equipped with UV absorbance optics. Two different methodological variants, the sedimentation equilibrium as well as sedimentation velocity techniques were used.

Sedimentation equilibrium experiments were analyzed using externally loaded six-channel cells with $12 \mathrm{~mm}$ optical path length and the capacity to handle three solvent solution pairs of $\sim 70 \mu$ liquid. Sedimentation equilibrium was reached after $2 \mathrm{~h}$ of over speed at 20,000 rpm, followed by an equilibrium speed of $16,000 \mathrm{rpm}$ for $\sim 30 \mathrm{~h}$ at $10^{\circ} \mathrm{C}$. Depending on the loading concentration the radial absorbance in each compartment was recorded at three different wavelengths between 260 and $290 \mathrm{~nm}$ using the molar absorbance coefficients. Molecular mass calculations employed the global fit of three radial distribution curves described by $A_{r}=A_{r m}$ eMF with $F=[(1-$ $\left.\rho v) \omega^{2}\left(r^{2}-r^{2}\right)\right] /(2 R T)$ using our program POLYMOLE [6]. In these equations, $A_{r}$ is the radial absorbance, $A_{r m}$ is the corresponding absorbance at meniscus position, $\rho$ is the solvent density, $v$ is the partial specific volume, $\omega$ is the angular velocity, $R$ is the gas constant, and $T$ is the absolute temperature, $M$ is the molecular mass and $\mathrm{rm}$ is the radius at meniscus point.

Sedimentation velocity runs were performed to estimate sedimentation (S) and diffusion coefficients (D) for the direct calculation of the molecular mass. 
About $400 \mu \mathrm{l}$ solute were filled into standard double sector cells and centrifuged at 50,000 rpm. Sedimentation and diffusion coefficients were determined from the time dependent records of the whole boundaries using our program LAMM [6]. From sedimentation and diffusion coefficients as well as the partial specific volume $(v)$ the molecular masses of the lever arms were determined by the Svedberg equation. Furthermore, these parameters allowed calculation of the frictional ratio $\left(f / f_{0}\right)_{\text {total }}=10-8[(1-v \rho) /(\mathrm{sD} v)] 1 / 3$ which gives an estimate of the gross conformation, where $v$ is the partial specific volume, and $\rho$ is the solvent density. The frictional ratio is influenced by two unknown variables, that is shape and hydration. Assuming an average hydration of about $0.3 \mathrm{~g}$ of bound water per $1.0 \mathrm{~g}$ of peptide, a hydrationdependent fraction of $\left(f / f_{0}\right)_{\text {hy }} \approx 1.13$ is obtained using $\left.\left(f / f_{0}\right)_{\text {hy }}=10-8(1-\mathrm{w}) / v \rho\right) 1 / 3$ where $w$ is the portion of bound water. The total value $\left(f / f_{0}\right)_{\text {total }}$ was thus divided by the factor 1.13 to obtain the shape-dependent frictional ratio. The latter allowed us to estimate the molecular dimensions. Knowledge of $\mathrm{M}$ and $v$ allows the calculation of the dry volume $V=[\mathrm{Mv}] / \mathrm{NA}$ with $\mathrm{NA}$ being the Avogadro number. Considering sphere-like molecules the dry volume can be described also by $V=1.33 \pi \cdot r^{3}$ with $r$ being the particle radius that corresponds to $r=[0.75 \mathrm{~V} / \mathrm{m}]^{1 / 3}$. In case of prolate ellipsoids of revolution with the half axes a and $b(a>b) \vee$ can be expressed $V=1.33 \pi a b^{2}$. Based on these relations the dimensions of the recombinant lever arms can be estimated. 
Circular dichroism spectroscopy

Circular dichroism (CD) spectra of His-MLC-1, GST-MYH ${ }_{664-915}$ and GST$\mathrm{MYH}_{664-915}{ }^{\text {IQala4 }}$ fusion proteins were recorded in a $1 \mathrm{~mm}$ quartz cuvette (Hellma, Müllheim, Germany) on a J-720 spectrometer (Jasco, Tokyo, Japan) at $25^{\circ} \mathrm{C}$ using a scanning speed of $50 \mathrm{~nm} / \mathrm{min}$, a bandwidth of $1 \mathrm{~nm}$, and a response time of $2 \mathrm{~s}$. Proteins were dissolved at concentrations of 4 or $6 \mu \mathrm{M}$ in $10 \mathrm{mM}$ Tris, $120 \mathrm{mM} \mathrm{NaF}, \mathrm{pH}$ 7.4. Presented spectra give the mean residual molar ellipticity $[\Theta]$ of one out of four independent experiments. Secondary structure compositions were estimated by deconvoluting CD spectra in the range of $205-240 \mathrm{~nm}$ [7] into reference spectra obtained from proteins of known structures.

\section{Statistics}

Statistical difference between mean values were calculated using Student's $t$ test for unpaired or paired values and were considered significant at $p$-values of $<0.05$. 


\section{Results and Discussion}

Besides its role as a protein recognition motif, the IQ2 motif exerts an autoinhibitory action on myosin II function. Thus, deletion of IQ2 increased significantly myosin ATPase activity and could rescue both defective dictyostelium cells with targeted MYH [8] as well as defective Schizosaccharomyces cells with targeted RLC [9]. In this study, we elaborated a structural explanation for this auto-inhibitory effect of IQ2 on myosin function and cell survival.

We produced fusion proteins, namely: (1) a rat GST-MYH7 head-rod fragment aa664-915 (GST-MYH $664-915$ ), i.e. the $20 \mathrm{kDa}$ S1-fragment (aa664-837; comprising the $\mathrm{SH} 1 / \mathrm{SH} 2$ helices with the two reactive cysteines $\mathrm{C} 697$ and C707, the converter domain from aa714-770, and the $8.5 \mathrm{~nm}$ a-helical lever arm from aa771-837 with the MLC binding IQ motifs), as well as a part of the a-helical myosin-S2 domain from aa838-915, and (2) rat ventricular Histagged MLC-1 (His-MLC-1).

His-MLC-1 revealed a high degree of a-helicity in the CD spectrum (Fig. 2) which is in accordance with the known EF-hand structure of the MLC [2-5]. Fitted CD spectra of the head-rod fragments show mainly a-helical secondary structures with around $50 \%$ a-helicity of the head-rod constructs (Fig. 2). Molecular mass of the GST-MYH $\mathrm{M}_{64-915}$ was found to be about $57 \mathrm{kDa}$, similar to the prediction from the protein sequence (Table 1). GST-MYH $\mathrm{H64-915}_{\text {exists }}$ as monomer in dilute solution, as shown by sedimentation equilibrium ultracentrifugation (Fig. 2). This is surprising, since GST-MYH $664-915$ contains 78aa of the a-helical myosin-S2 domain, which could form a coiled-coil due to the presence of characteristic heptad sequence repeats. A recently analyzed 
126aa $\beta$-myosin-S2 segment (aa838-963) forms a straight parallel coiled-coil in the crystal structure [10]. However, the N-terminus of myosin-S2 appeared disordered rather than $\alpha$-helical [10], so that our head-rod fragment may not reach the critical $\alpha$-helical rod length required for self-assembly into a coiledcoil. Fragments of scallop MYH composed of the MLC binding domain and a rod extension of 87aa remained monomeric [11] while an N-terminal 150aa fragment of smooth muscle-S2 fails to fully dimerize [12]. We observed indeed a tendency to dimerization of our head-rod proteins, but only above $5 \mu \mathrm{M}$ (Fig. 2), i.e. concentrations above those used in the experiments described herein. GST-MYH $\mathrm{H}_{64-915}$ adopts a sphere-like shape, as evidenced by a small frictional ratio of $f / f_{0}=1.08$. We calculated a total length of GST-MYH $\mathrm{H}_{664-915}$ of around $10.4 \mathrm{~nm}$ with a mean diameter of the molecules of ca. $3.6 \mathrm{~nm}$ (calculated from the axial ratio of a hydrodynamically equivalent prolate ellipsoid of revolution; (Table 1, Figure 3). Albeit the available crystal structures of myosin-S1 and myosin-S2 represent stretched and rigid structures (c.f. Figure 3), the observed short and compact shape of the head-rod fragment observed herein is not unexpected. It is a remarkable property of all type II myosin isoforms to fold [13] into a compact form, which increases the sedimentation coefficient from $6 \mathrm{~S}$ (typical for extended myosin) to $10 \mathrm{~S}$, which is of importance for myosin function and in particular for smooth muscle contraction [14],. The unstable head-rod-junction seems to promote folding into the compact conformation: the $\mathrm{N}$-terminus of myosin-S2 appeared disordered $[10,15]$ and may serve as a skip domain for myosin bending. In the crystal structure of myosin-S2 (2fxo), the N-terminus do not form a coiled-coil since a polar contact between E916 from a neighboring molecule and the M852 disrupts 
the $\mathrm{N}$-terminus, leading to the absence of coiled-coil interactions and disorder of the first 12aa residues [10]. A recent atomic model which includes the two heads and two myosin-S2 segments [16] demonstrates the kink between myosin-S1 and the N-terminus of a myosin-S2 fragment even in the intact thick filament (c.f. also [17]).

To investigate if the presence of the IQ2 domain could also determine the structure of the MYH, we expressed an IQ2 deletion mutation of the head-rod domain (GST-MYH ${ }_{664-915}{ }^{\Delta \mathrm{IQ} 2}$ ). The molecular mass of GST-MYH ${ }_{664-915}{ }^{\Delta \mathrm{Q} 2}$ was 55.7k Da, and exists as monomer in dilute solution. Importantly, GST-MYH $664-$ $915^{\Delta \mathrm{IQ} 2}$ revealed an open structure with $f / f_{0}$ of 1.49 which corresponds well to the predicted conformation of the available 3D-crystal structures. Total length of GST-MYH ${ }_{664-915}{ }^{\Delta \mathrm{QQ} 2}$ was around $21.2 \mathrm{~nm}$ with mean diameter of ca. $2.5 \mathrm{~nm}$ (Table 1, Figure 3).

Four amino acids present in the IQ2 motif could be identified to be responsible for the auto-inhibitory folding effect of IQ2 on protein structure by alanine substitutions, namely 1814, Q815, R819, and W827, i.e. key amino acids involved in RLC binding [4, 5]. Similar to $\mathrm{MYH}_{664-915}$, the molecular mass of the alanine mutated (GST-MYH ${ }_{664-915}{ }^{\text {IQ2ala4}}$ ) head-rod fragment was about 57 $\mathrm{kDa}$ and exists as monomer in diluted solution. GST-MYH $\mathrm{H}_{664-915}{ }^{\text {IQ2ala4 }}$ revealed an open structure, like the GST- GST-MYH ${ }_{664-915}{ }^{\Delta \mathrm{QQ} 2}$ fragment, with $f / f_{0}$ of 1.51 (Table 1). Total length of GST-MYH ${ }_{664-915}{ }^{\mathrm{IQ} 2}$ was around $22 \mathrm{~nm}$ with mean diameter of ca. $2.4 \mathrm{~nm}$ (Table 1, Figure 3) and 70\% $\alpha$-helicity.

The distinct conformations of the head-rod fragments predict different functional properties. To test this hypothesis, SPR analysis of the interaction between HIS-MLC-1 and IQ1 of GST-MYH ${ }_{664-915}$ and GST-MYH ${ }_{664-915}^{\text {IQ2ala4, }}$ 
respectively, i.e. the closed and open forms, were investigated. The GST$\mathrm{MYH}_{664-915} /$ His-MLC-1 complex revealed weak binding with a dissociation constant $\left(\mathrm{K}_{\mathrm{D}}\right)$ of around $1 \mu \mathrm{M}$ (Table 2, Fig. 4). A simple explanation for this weak ELC interaction could be a sterical hindrance of IQ1 in the closed headrod fragment (Fig. 3). In contrast, binding properties of the open head-rod fragment improved considerably: the $\mathrm{K}_{\mathrm{D}}$ of the GST-MYH ${ }_{664-915}{ }^{\text {IQ2ala4} / H i s-M L C-~}$ 1 complex dropped significantly $(p<0.001)$ almost 30 -fold compared with the closed GST-MYH $664-915 /$ His-MLC-1complex (Table 2). This pronounced increase in binding affinity of His-MLC-1 to IQ1 could be attributed to a conspicuous 70 -fold increase of $k_{o n}(p<0.001)$ (Table 2).

\section{Conclusions}

The presence of the IQ2 motif could strongly influence the conformation of adjacent protein domains and, therefore function of neighboring protein interaction motifs. Our new findings of the auto-inhibitory effect of IQ2 on myosin structure and its functional consequences on MLC-1 binding could provide a reasonable explanation for the auto-inhibitory action of IQ2 on myosin functions: the denuded IQ2 motif enforces a collapsed head-rod structure of myosin II, and subsequently hinders binding of ELC to IQ1, a critical interaction for full force generation of the lever arm $[8,18]$ and, therefore normal cellular functions. It remains to be elucidated, if such an auto-inhibitory structural effect on adjacent protein structures is restricted to incomplete IQ motifs. 


\section{Acknowledgements}

We thank Dr. J.J. Müller (MDC, Berlin, Germany) for preparing the head-rod schemes. This work was supported by DFG grant Mo 362/25-1 for IM.

\section{References}

[1] A. R. Rhoads, and F. Friedberg, Sequence motifs for calmodulin recognition, Faseb J 11 (1997) 331-340.

[2] I. Rayment, H. M. Holden, M. Whittaker, C. B. Yohn, M. Lorenz, K. C. Holmes, and R. A. Milligan, Structure of the actin-myosin complex and its implications for muscle contraction, Science 261 (1993) 58-65.

[3] I. Rayment, W. R. Rypniewski, K. Schmidt-Base, R. Smith, D. R. Tomchick, M. M. Benning, D. A. Winkelmann, G. Wesenberg, and H. M. Holden, Three-dimensional structure of myosin subfragment-1: a molecular motor, Science 261 (1993) 50-58.

[4] A. Houdusse, and C. Cohen, Target sequence recognition by the calmodulin superfamily: implications from light chain binding to the regulatory domain of scallop myosin, Proc Natl Acad Sci U S A 92 (1995) 10644-10647.

[5] X. Xie, D. H. Harrison, I. Schlichting, R. M. Sweet, V. N. Kalabokis, A. G. Szent-Gyorgyi, and C. Cohen, Structure of the regulatory domain of scallop myosin at 2.8 A resolution, Nature 368 (1994) 306-312.

[6] J. Behlke, and O. Ristau, Sedimentation equilibrium: a valuable tool to study homologous and heterogeneous interactions of proteins or proteins and nucleic acids, Eur Biophys J 32 (2003) 427-431.

[7] Y. H. Chen, J. T. Yang, and H. M. Martinez, Determination of the secondary structures of proteins by circular dichroism and optical rotatory dispersion, Biochemistry 11 (1972) 4120-4131.

[8] T. Q. Uyeda, and J. A. Spudich, A functional recombinant myosin II lacking a regulatory light chain-binding site, Science 262 (1993) 18671870. 
[9] N. I. Naqvi, K. C. Wong, X. Tang, and M. K. Balasubramanian, Type II myosin regulatory light chain relieves auto-inhibition of myosin-heavychain function, Nat Cell Biol 2 (2000) 855-858.

[10] W. Blankenfeldt, N. H. Thoma, J. S. Wray, M. Gautel, and I. Schlichting, Crystal structures of human cardiac beta-myosin II S2Delta provide insight into the functional role of the S2 subfragment, Proc Natl Acad Sci U S A 103 (2006) 17713-17717.

[11] A. Malnasi-Csizmadia, E. Shimony, G. Hegyi, A. G. Szent-Gyorgyi, and L. Nyitray, Dimerization of the head-rod junction of scallop myosin, Biochem Biophys Res Commun 252 (1998) 595-601.

[12] K. M. Trybus, Regulation of expressed truncated smooth muscle myosins. Role of the essential light chain and tail length, J Biol Chem 269 (1994) 20819-20822.

[13] H. S. Jung, S. Komatsu, M. Ikebe, and R. Craig, Head-head and headtail interaction: a general mechanism for switching off myosin II activity in cells, Mol Biol Cell 19 (2008) 3234-3242.

[14] K. M. Trybus, Myosin regulation and assembly, ed., Barany Med. Academic press 1996.

[15] Y. Li, J. H. Brown, L. Reshetnikova, A. Blazsek, L. Farkas, L. Nyitray, and $\mathrm{C}$. Cohen, Visualization of an unstable coiled coil from the scallop myosin rod, Nature 424 (2003) 341-345.

[16] J. L. Woodhead, F. Q. Zhao, R. Craig, E. H. Egelman, L. Alamo, and R. Padron, Atomic model of a myosin filament in the relaxed state, Nature 436 (2005) 1195-1199.

[17] J. H. Brown, Y. Yang, L. Reshetnikova, S. Gourinath, D. Suveges, J. Kardos, F. Hobor, R. Reutzel, L. Nyitray, and C. Cohen, An unstable head-rod junction may promote folding into the compact off-state conformation of regulated myosins, J Mol Biol 375 (2008) 1434-1443.

[18] P. VanBuren, G. S. Waller, D. E. Harris, K. M. Trybus, D. M. Warshaw, and S. Lowey, The essential light chain is required for full force production by skeletal muscle myosin, Proc Natl Acad Sci U S A 91 (1994) 12403-12407. 


\section{Figure captions}

Fig. 1. Schematic diagram of myosin II in an open conformation. Myosin II consists of two heavy chains each consisting of a subfragment-1 (S1), a S2, and light meromyosin (LMM). S1 comprises the motor domain (MD) and the regulatory domain (RD) associated with an essential (ELC) and a regulatory (RLC) light chain. The spotted box in S2 indicates the disordered region immediately adjacent to the head-rod junction (adapted from $[16,17]$ )

Fig. 2. Structural analysis of the myosin head-rod and myosin light chain fusion proteins. A) Circular dichroism spectra (calculated fitted curve) of

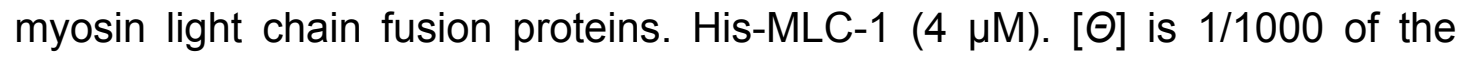
mean residual molar ellipticity. Noisy curve represents original signals, smooth curve represents the corresponding calculated fit. B) Circular dichroism spectra of head-rod fusion proteins (calculated fitted curves). Grey: GST-MYH $_{664-915}{ }^{\text {IQ2ala4}}$; black: GST-MYH ${ }_{664-915}(4 \mu \mathrm{M}$ each). [ $\Theta]$ is $1 / 1000$ of the mean residual molar ellipticity. Noisy curve represents original signals, smooth curve represents the corresponding calculated fits C) Sedimentation equilibrium analysis of head-rod fragments (1.0-6.5 $\mu \mathrm{M})$. Open squares: GST$\mathrm{MYH}_{664-915}$; closed squares: GST-MYH ${ }_{664-915}{ }^{\text {IQ2ala4 }}$.

Fig. 3. Schemes of monomeric head-rod fragments based on available 3Dstructures mounted according to data obtained by analytical ultracentrifugation. A) Scheme of $\mathrm{MYH}_{664-915}$ assembled from three available crystal structures: GST (PDB 2GST; in blue), chicken skeletal muscle myosinS1 (aa664-843; PDB 2MYS; in green), and a single strand of the (originally 
coiled-coil) myosin-S2 (rod)-fragment (aa849-920; PDB 2fxo; in red) using Pymol.. In this scheme, all three head-rod segments in a stretched conformation would yield a length of around $20 \mathrm{~nm}$. B) Scheme of $\mathrm{MYH}_{664-915}$ assembled to fit the ultracentrifugation velocity data: the head-rod fragment revealed a compact conformation $\left(f / f_{0}=1.08\right.$, Table1) with a large axis of only $10.4 \mathrm{~nm}$, i.e. the just length of the $20 \mathrm{kDa}$ S1-fragment (c.f crystal structure 2MYS). In analogy to the structural myosin model of Woodhead et al., 2005 [14],we then assumed a bending point at the unstructured head-rod junction [10] (arrow), an intervention which finally met the ultracentrifugation data. (Table1). C): Proposed scheme of GST-MYH ${ }_{664-915}{ }^{\Delta I Q 2}$ or GST-MYH $664-$ $915^{\text {IQ2ala4 }}$, the IQ2 deleted and alanine-mutated GST-MYH $664-915$ variants. Data from analytical ultracentrifugation predicts an open conformations with large axis of around $22 \mathrm{~nm}$, i.e. a length which could be obtained if all three headrod elements are connected in series. Illustrations were prepared using UCSF Chimera.

Fig. 4. Protein analysis and protein-protein interactions. A) SDS-PAGE of HisMLC-1 (1), GST-MYH $\mathrm{H}_{664-915}$ (2) and GST-MYH G64-915 $^{\text {IQ2ala4 }}$ (3) B) Representative original registration of surface plasmon resonance signals of the interactions between His-MLC-1/GST-MYH $664-915$ (grey line) and His-MLC1/GST-MYH ${ }_{664-915}{ }^{\text {IQ2ala4 }}$ (black line). 


\section{Tables}

Table 1. Sedimentation velocity analysis of GST-MYH $\mathrm{H}_{664-915}$ and its alaninescanning mutated derivative GST-MYH ${ }_{664-915}{ }^{\text {IQ2ala4 }}$. s20,w: Sedimentation coefficient in Svedberg Units; D20,w: diffusion coefficient; v: partial specific volume; M: molar mass; $\left(f / f_{0}\right)_{\text {total: }}$ frictional ratio (reflects the shape of the molecule); $\left(f / f_{0}\right)$ shape: frictional ratio considering $0.3 \mathrm{~g}$ water/g protein; V: dry volume; a/b: axial ratio of the hydrodynamically equivalent prolate ellipsoid of revolution; a: large half axis; b: small half axis.

Table 2. Surface plasmone resonance analysis between GST-MYH $664-915$ and GST-MYH ${ }_{664-915}{ }^{\text {IQ2ala4 }}$, respectively and the rat ventricular myosin light chain. Interactions between wild-type (GST-MYH $\left.{ }_{664-915}\right)$ and alanine-mutated (GST-

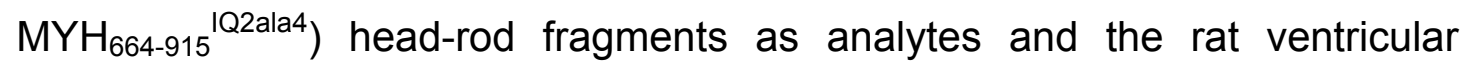
myosin light chain (His-MLC-1) as ligand. $\mathrm{k}_{\text {on }}$ : association rate constant; $\mathrm{k}_{\text {off: }}$ dissociation rate constant; $K_{D}=$ dissociation constant. $\mathrm{n}$ is the number of experiments. ${ }^{* * *} \mathrm{p}<0.001$ versus GST-MYH $664-915$. 


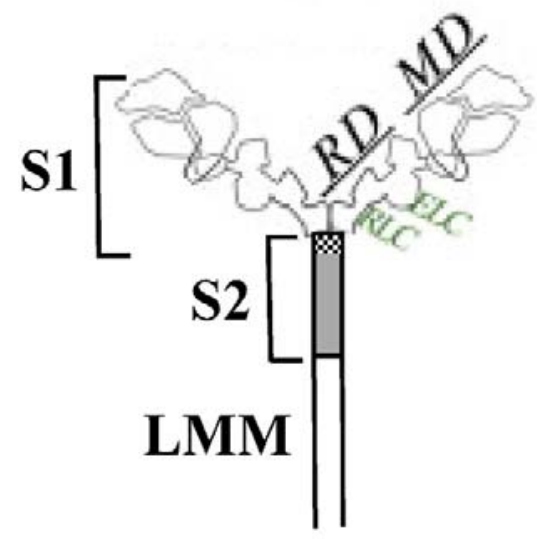

Figure 1
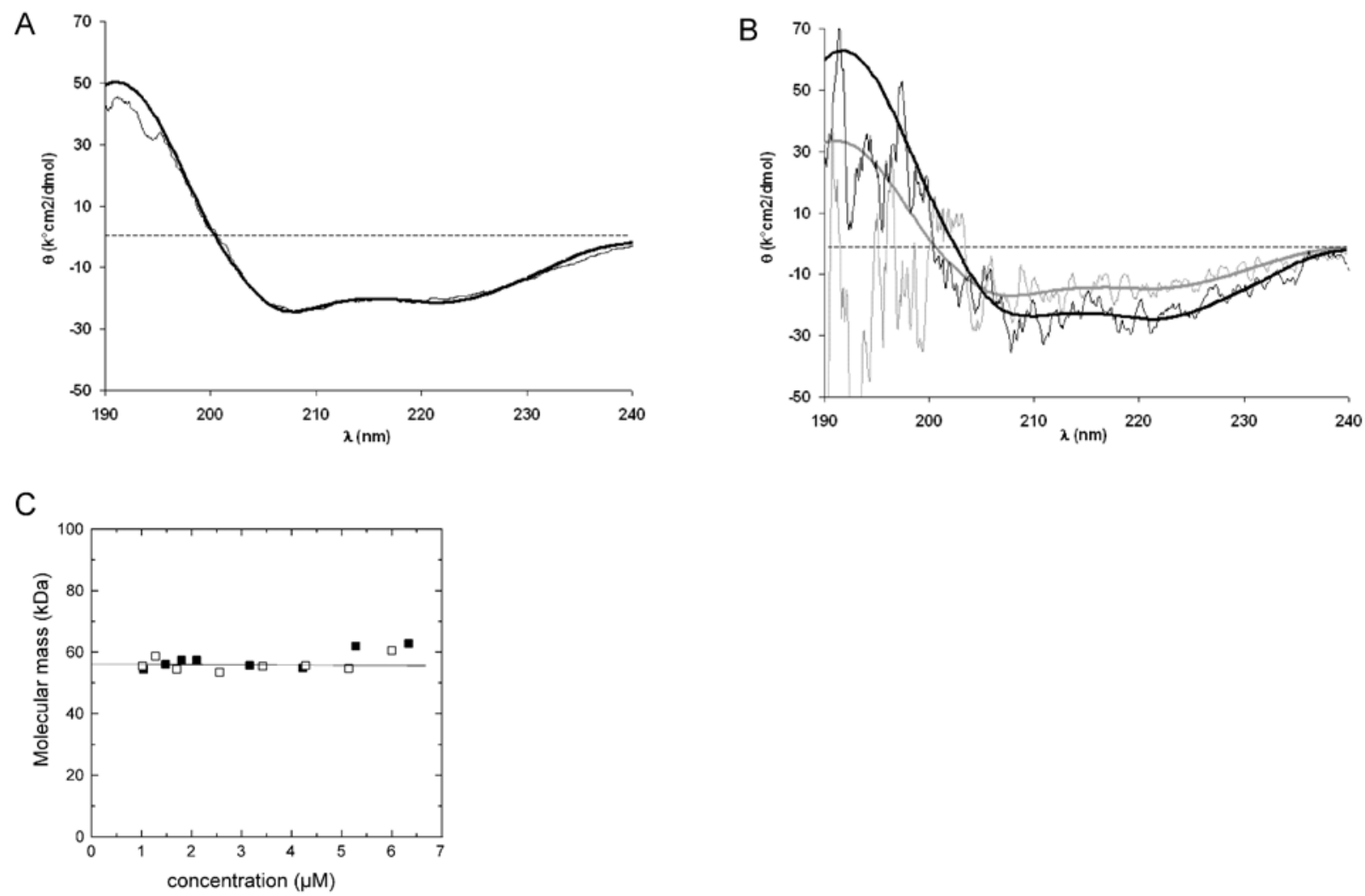

Figure 2 
A

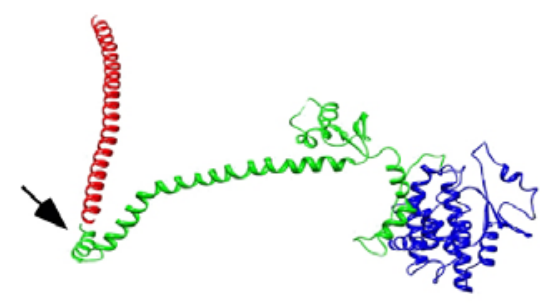

B

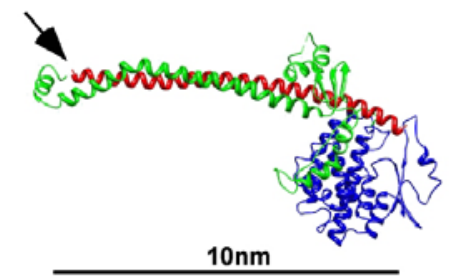

C

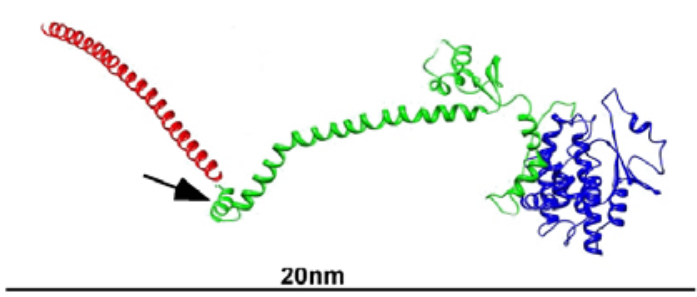

Figure 3

A

$\mathrm{kDa}$

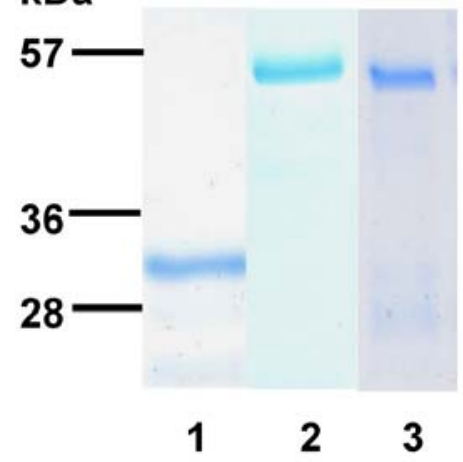

B

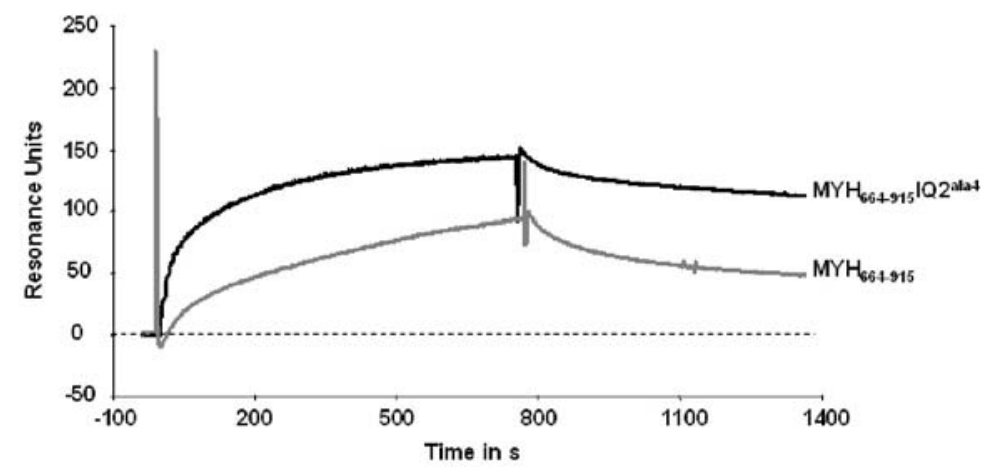

Figure 4 


\begin{tabular}{|c|c|c|c|}
\hline & $\mathrm{MYH}_{664-915}$ & $\mathrm{MYH}_{664-915}{ }^{\Delta \mathrm{QQ2}}$ & $\begin{array}{l}\mathbf{M Y H}_{664-915} \mathrm{IQ2}^{\text {ala4 }} \\
271\end{array}$ \\
\hline $\begin{array}{l}S_{20, w}(S) \\
D_{20, w}\left(10^{7} \mathrm{~cm}^{2} / \mathrm{s}\right)\end{array}$ & $\begin{array}{l}3.86 \\
6.81\end{array}$ & $\begin{array}{l}2.84 \\
4.97\end{array}$ & $\begin{array}{l}2 . / 1 \\
4.76\end{array}$ \\
\hline $\mathrm{v}(\mathrm{ml} / \mathrm{g})$ & 0.75 & 0.75 & 0.75 \\
\hline M (kDa) & 57.0 & 55.7 & 57.0 \\
\hline$\left(f / f_{0}\right)_{\text {total }}$ & 1.22 & 1.68 & 1.71 \\
\hline$\left(f / f_{0}\right)_{\text {shape }}$ & 1.08 & 1.49 & 1.51 \\
\hline$a / b$ & $\sim 2.8$ & $\sim 8.5$ & $\sim 9$ \\
\hline$a(n m)$ & $\sim 5.2$ & 10.6 & $\sim 11$ \\
\hline b (nm) & $\sim 1.8$ & $\sim 1.25$ & $\sim 1.2$ \\
\hline
\end{tabular}

Table 1

$\begin{array}{lcccr}\text { Analyte } & \mathbf{k}_{\text {on }}\left(\mathbf{M}^{-1} \mathbf{s}^{-1}\right) & \mathbf{k}_{\text {off }}\left(\mathbf{s}^{-1}\right) & \mathbf{K}_{\mathrm{D}}(\mathbf{n M}) & \mathbf{n} \\ \text { MYH }_{664-915} & 518.8 \pm 48.9 & 5.3^{*} 10^{-4} \pm 0.8^{*} 10^{-4} & 1101.5 \pm 214.7 & 10 \\ \text { MYH }_{664-915} \mathbf{I Q 2}^{\text {ala4 }} & 3.6^{*} 10^{4} \pm 0.8^{*} 10^{4 * * *} & 7.3^{*} 10^{-4} \pm 1,8^{*} 10^{-4^{* *}} & 34.8 \pm 8.0^{\star \star *} & 12\end{array}$

Table 2 